\title{
The lifetimes of evaporating sessile droplets are significantly extended by strong thermal effects
}

\author{
F. G. H. Schofield ${ }^{1}$, S. K. Wilson ${ }^{1} \dagger$, D. Pritchard ${ }^{1}$ and K. Sefiane ${ }^{2}$ \\ ${ }^{1}$ Department of Mathematics and Statistics, University of Strathclyde, Livingstone Tower, \\ 26 Richmond Street, Glasgow G1 1XH, Scotland, UK \\ ${ }^{2}$ School of Engineering, University of Edinburgh, The King's Buildings, Mayfield Road, \\ Edinburgh EH9 3JL, Scotland, UK
}

(Received 14 November 2017; revised 20 May 2018; accepted 13 June 2018;

first published online 19 July 2018)

The evaporation of sessile droplets is analysed when the influence of the thermal properties of the system is strong. We obtain asymptotic solutions for the evolution, and hence explicit expressions for the lifetimes, of droplets when the substrate has a high thermal resistance relative to the droplet and when the saturation concentration of the vapour depends strongly on temperature. In both situations we find that the lifetimes of the droplets are significantly extended relative to those when thermal effects are weak.

Key words: condensation/evaporation, phase change, thin films

\section{Introduction}

The evaporation of sessile droplets has been the subject of increasing theoretical and experimental research activity in recent years (see, for example, Cazabat \& Guéna 2010, Erbil 2012, Larson 2014 and Lohse \& Zhang 2015). In particular, there is growing interest in how the evolution, and hence the lifetimes, of evaporating droplets depend on the thermal properties of the system.

In their pioneering work on the evaporation of sessile droplets, Picknett \& Bexon (1977) calculated the lifetimes of droplets evaporating in the so-called extreme modes of evaporation, namely the constant contact radius (CR) and constant contact angle (CA) modes. In practice, however, droplets often evaporate in the so-called stick-slide (SS) mode consisting of a CR phase followed by a CA phase (see, for example, Nguyen \& Nguyen 2012 and Dash \& Garimella 2013), and so Stauber et al. (2014, 2015) calculated the lifetime of a droplet evaporating in this mode. Other modes of evaporation can, of course, also occur, notably the so-called stick-jump (SJ) mode in which the droplet evaporates in a series of stick (i.e. CR) phases separated by jump phases in which the contact angle and contact radius jump instantaneously (see, for example, Askounis et al. 2011, Orejon, Sefiane and Shanahan 2011 and Dietrich

$\dagger$ Email address for correspondence: s.k.wilson@strath.ac.uk 


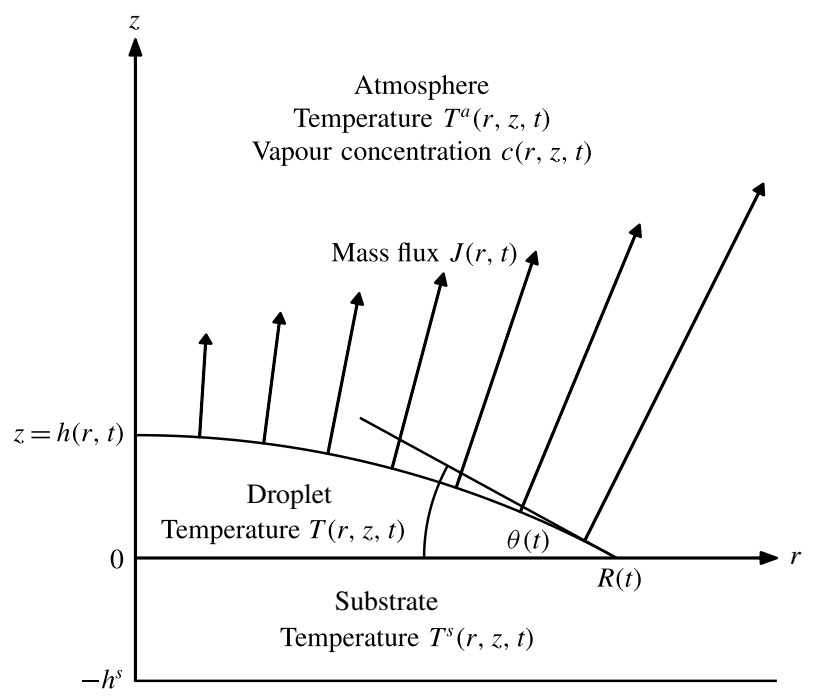

FIGURE 1. The geometry of a evaporating thin sessile droplet on a thin substrate.

et al. 2015), and so Stauber (2015) performed the corresponding analysis of a droplet evaporating in this mode. However, all of these works use the basic diffusion-limited model for droplet evaporation (see, for example, Popov 2005) which does not account for the variation of the saturation concentration of the vapour with temperature. As a result, none of these works accounts for the influence of the thermal properties of the system on the evaporation of the droplets, which can be significant (see, for example, Dunn et al. 2008, 2009a,b, Sefiane \& Bennacer 2011, Sobac \& Brutin 2012, Ait Saada, Chikh and Tadrist 2013, Lopes et al. 2013 and Diddens et al. 2017). The aim of the present work is to gain further insight into this issue by using an extended model to obtain asymptotic solutions for the evolution, and hence explicit expressions for the lifetimes, of droplets when the influence of the thermal properties of the system is strong.

\section{Problem formulation}

Consider the quasi-steady evaporation of a small thin axisymmetric sessile droplet with radius $R=R(t)$, contact angle $\theta=\theta(t)$ and volume $V=V(t)$ of a fluid with constant surface tension $\sigma$, density $\rho$ and thermal conductivity $k$ on a thin substrate with constant thickness $h^{s}$ and thermal conductivity $k^{s}$ in a quiescent atmosphere with thermal conductivity $k^{a}$. Using cylindrical polar coordinates $(r, z)$ with their origin on the substrate at the centre of the droplet, the free surface of the droplet is denoted by $z=h(r, t)$, the concentration of vapour in the atmosphere is denoted by $c=c(r, z, t)$ and the temperatures of the atmosphere, the droplet and the substrate are denoted by $T^{a}=T^{a}(r, z, t), T=T(r, z, t)$ and $T^{s}=T^{s}(r, z, t)$, respectively. The geometry of the problem is shown in figure 1.

The transport of vapour in the atmosphere is due solely to diffusion, and so $c$ satisfies Laplace's equation in the appropriate domain. At the free surface of the droplet the atmosphere is saturated with vapour, and so $c$ takes its saturation value, denoted by $c_{s a t}=c_{s a t}(T)$, which we assume to be a linearly increasing function of $T$ given by $c_{\text {sat }}(T)=c_{s a t}\left(T_{\infty}\right)+c_{s a t}^{\prime}\left(T_{\infty}\right)\left(T-T_{\infty}\right)$. (Note that Sefiane et al. (2009) and 
Ait Saada et al. (2013) considered a nonlinear dependence of $c_{\text {sat }}$ on $T$.) There is no mass flux through the unwetted part of the substrate, and far from the droplet $c$ approaches its ambient value $H c_{\text {sat }}\left(T_{\infty}\right)$, where $H(0 \leqslant H \leqslant 1)$ denotes the relative saturation of the ambient atmosphere. The local evaporative mass flux from the droplet, denoted by $J=J(r, t)$, is given by $J=-D \nabla c \cdot \boldsymbol{n}$, where $D$ is the coefficient of diffusion of vapour in the atmosphere and $\boldsymbol{n}$ is the unit outward normal to the free surface of the droplet. The total evaporation rate, $-\rho \mathrm{d} V / \mathrm{d} t$, is obtained by integrating $J$ over the free surface of the droplet.

Transport of heat in the atmosphere, the droplet and the substrate is due solely to thermal conduction, and so $T^{a}, T$ and $T^{s}$ also satisfy Laplace's equation in the appropriate domains. At the free surface of the droplet $J$ satisfies the local energy balance

$$
\mathcal{L} J=\boldsymbol{n} \cdot\left(k^{a} \nabla T^{a}-k \nabla T\right),
$$

where $\mathcal{L}$ is the latent heat of vaporisation, while on both the wetted and unwetted parts of the substrate both the temperature and the heat flux are continuous, and far from the droplet $T^{a}$ approaches its ambient value $T_{\infty}$. Note that if we were simply to set $T^{a} \equiv T_{\infty}$ (which does not, in general, satisfy the conditions of continuity of heat flux on the unwetted part of the substrate or of continuity of temperature on the free surface of the droplet) we would recover the extended model proposed by Dunn et al. $(2009 a)$. However, as described below, in the present work we exploit the fact that in practice the atmosphere is typically a relatively poor thermal conductor to obtain a more physically realistic extended model.

In order to make analytical progress we follow Dunn et al. (2008, 2009b) and assume that both the droplet and the substrate are thin (i.e. that $\theta(0) \ll 1$ and $\left.h^{s} / R(0) \ll 1\right)$, but make no assumption about their relative thicknesses (i.e. we make no a priori assumption about the size of $\left.h^{s} /(\theta(0) R(0))\right)$. Hence at leading order in the appropriate thin-film limit, the free surface of the droplet is given by $h=\theta\left(R^{2}-r^{2}\right) /(2 R)$ and the volume of the droplet is given by $V=\pi \theta R^{3} / 4$.

We non-dimensionalise and scale $r$ with $R(0), z$ in the droplet with $\theta(0) R(0), z$ in the substrate with $h^{s}, z$ in the atmosphere with $R(0), h$ with $\theta(0) R(0), V$ with $\theta(0) R(0)^{3}, \theta$ with $\theta(0), T^{a}, T$ and $T^{s}$ with $T_{\infty}, c-H c_{\text {sat }}\left(T_{\infty}\right)$ with $(1-H) c_{\text {sat }}\left(T_{\infty}\right), J$ with $D(1-H) c_{s a t}\left(T_{\infty}\right) / R(0)$ and $t$ with the characteristic time scale for the evaporation of a thin droplet according to the basic model, namely

$$
\frac{\rho \theta(0) R(0)^{2}}{D(1-H) c_{\text {sat }}\left(T_{\infty}\right)} .
$$

In practice, the atmosphere is typically a relatively poor thermal conductor, and provided that

$$
k^{a} \ll \frac{k}{\theta(0)} \quad \text { and } \quad k^{a} \ll \frac{R(0) k^{s}}{h^{s}}
$$

the problem for $T^{a}$ decouples from that for $T, T^{s}$ and $c$. Note that, since both the droplet and the substrate are thin, these conditions hold when $k$ and $k^{s}$ are comparable with $k^{a}$. When (2.3) holds it is straightforward to show that $T$ and $T^{s}$ are given by

$$
\begin{gathered}
T=1-E J(z+S) \text { for } 0<z<h, r<R, \\
T^{s}=1-\operatorname{EJS}(z+1) \text { for }-1<z<0, r<R, \\
T^{s}=1 \text { for }-1<z<0, r>R,
\end{gathered}
$$


that $c$ satisfies

$$
\nabla^{2} c=0 \quad \text { in } z>0
$$

subject to

$$
\begin{gathered}
c=1+\Delta C(h+S) \frac{\partial c}{\partial z} \quad \text { on } z=0 \text { for } r<R, \\
\frac{\partial c}{\partial z}=0 \quad \text { on } z=0 \text { for } r>R,
\end{gathered}
$$

and

$$
c \rightarrow 0 \quad \text { as }\left(r^{2}+z^{2}\right)^{1 / 2} \rightarrow \infty
$$

and that $T^{a}$ satisfies

$$
\nabla^{2} T^{a}=0 \quad \text { in } z>0
$$

subject to

$$
\begin{gathered}
T^{a}=1-E J(h+S) \quad \text { on } z=0 \text { for } r<R, \\
T^{a}=1 \quad \text { on } z=0 \text { for } r>R,
\end{gathered}
$$

and

$$
T^{a} \rightarrow 1 \quad \text { as }\left(r^{2}+z^{2}\right)^{1 / 2} \rightarrow \infty
$$

The total evaporation rate is then given by

$$
-\frac{\mathrm{d} V}{\mathrm{~d} t}=-\frac{\pi}{4} \frac{\mathrm{d}}{\mathrm{d} t}\left(\theta R^{3}\right)=2 \pi \int_{0}^{R} J(r, t) r \mathrm{~d} r
$$

where

$$
J(r, t)=-\left.\frac{\partial c}{\partial z}\right|_{z=0}
$$

and

$$
\Delta C=\frac{\theta(0) \mathcal{L} D c_{s a t}^{\prime}\left(T_{\infty}\right)}{k}, \quad S=\frac{k h^{s}}{\theta(0) R(0) k^{s}} \quad \text { and } \quad E=\frac{\theta(0) \mathcal{L} D(1-H) c_{s a t}\left(T_{\infty}\right)}{k T_{\infty}}
$$

are non-dimensional measures of the variation of the saturation concentration of the vapour with temperature, the relative thermal resistance of the droplet and the substrate and the evaporative cooling, respectively. Whereas (2.7)-(2.10) show that the solution for $c$, and hence the evolution of the droplet given by (2.15) and (2.16), depends on $\Delta C$ and $S$ but not $E$, (2.4)-(2.6) and (2.11)-(2.14) show that the evaporative cooling of the atmosphere, the droplet and the substrate also depends on $E$.

Note that although, as we have already mentioned, the present extended model differs from that proposed by Dunn et al. (2009a), in the present limit of a thin droplet on a thin substrate the problem for $T, T^{s}$ and $c$ given by (2.4)-(2.10), but not, of course, that for $T^{a}$ given by (2.11)-(2.14), coincides exactly with that analysed by Dunn et al. $(2008,2009 b)$. However, these latter authors considered only the initial evolution of a pinned droplet (i.e. evolution of a droplet evaporating in the CR mode for $t=O(1)$ ) and did not analyse either the entire evolution of the droplet or consider other modes of evaporation, both of which we do in the present work. 
In the special case $\Delta C=0$ in which $c_{\text {sat }}$ is independent of $T$ the concentration of vapour at the free surface of the droplet is constant and we recover the basic model in which the solution for $c$, and hence the evolution of the droplet, is also independent of $S$, i.e. is entirely independent of the thermal properties of the system. Explicit expressions for the lifetimes of thin droplets in this special case (albeit using a different non-dimensionalisation of time) were obtained by Stauber et al. (2014) for the CR, CA and SS modes, and by Stauber (2015) for the SJ mode. For brevity, these expressions are not reproduced here, but the key observation is that when $\Delta C=0$ the lifetimes of thin droplets are, as anticipated, of the order of the basic time scale (2.2). This time scale is also applicable when thermal effects are weak, but not, as we shall show in the present work, when they are strong.

\section{When the substrate has a high thermal resistance}

Consider the evolution of an evaporating droplet using the extended model described in $\$ 2$ when the substrate has a high thermal resistance relative to the droplet (i.e. when the substrate is highly insulating and/or thick relative to the droplet), corresponding to the asymptotic limit $S \rightarrow \infty$ with $\Delta C \neq 0$.

Inspection of (2.7)-(2.15) suggests that in the limit $S \rightarrow \infty$ the complete evolution of a droplet will occur over the long time scale $t=O(S) \gg 1$. In order to obtain a uniformly valid leading-order asymptotic solution able to capture the complete evolution of the droplet we therefore rescale $c$ and $J$ with $1 / S$ and $t$ with $S$ by writing $c=\tilde{c} / S, J=\tilde{J} / S$ and $t=\tilde{S t}$, and seek an asymptotic expansion for $\tilde{c}$ in the form $\tilde{c}(r, z, \tilde{t})=\tilde{c}_{0}(r, z, \tilde{t})+O(1 / S)$ with corresponding asymptotic expansions for the other dependent variables.

At leading order in the limit $S \rightarrow \infty$ we obtain $\tilde{J}_{0}=1 / \Delta C$ (i.e. at leading order the mass flux from the free surface of the droplet is uniform and constant), $T_{0}=1-E / \Delta C$ (i.e. at leading order the droplet is uniformly cooled by a constant amount $E / \Delta C$ ) and $T_{0}^{s}=1-E(z+1) / \Delta C$ and hence (2.15) yields the equation describing the leading-order evolution of the droplet, namely

$$
\frac{\mathrm{d}}{\mathrm{d} \tilde{t}}\left(\theta_{0} R_{0}^{3}\right)=-\frac{4 R_{0}^{2}}{\Delta C} .
$$

An explicit expression for the leading-order concentration of vapour, $\tilde{c}_{0}$, can be obtained (see, for example, Mehta \& Bose 1983), but, since this is not required in order to determine the leading-order evolution of the droplet, it is not reproduced here for brevity.

\subsection{Evolution of a droplet evaporating in the constant radius mode}

For a droplet evaporating in the CR mode with $R_{0} \equiv 1$, solving (3.1) yields

$$
R_{0} \equiv 1, \quad \theta_{0}=1-\frac{4}{\Delta C} \tilde{t}, \quad V_{0}=\frac{\pi \theta_{0}}{4},
$$

and hence the lifetime of the droplet, denoted by $\tilde{t}_{C R}$, is given by $\tilde{t}_{C R}=\Delta C / 4$.

\subsection{Evolution of a droplet evaporating in the constant angle mode}

For a droplet evaporating in the CA mode with $\theta_{0} \equiv 1$, solving (3.1) yields

$$
R_{0}=1-\frac{4}{3 \Delta C} \tilde{t}, \quad \theta_{0} \equiv 1, \quad V_{0}=\frac{\pi R_{0}^{3}}{4}
$$

and hence the lifetime of the droplet, denoted by $\tilde{t}_{C A}$, is given by $\tilde{t}_{C A}=3 \Delta C / 4=3 \tilde{t}_{C R}$. 
Both $\tilde{t}_{C R}$ and $\tilde{t}_{C A}$ are directly proportional to $\Delta C$ (i.e. stronger variation of $c_{s a t}$ with $T$ leads to slower evaporation and hence to longer lifetimes), and their ratio is exactly 3. This latter result contrasts with that in the special case $\Delta C=0$ discussed by Stauber et al. (2014) for which the corresponding ratio is exactly $3 / 2$.

\subsection{Evolution of a droplet evaporating in the stick-slide mode}

The stick-slide (SS) mode of evaporation consists of a CR phase which lasts until the contact angle reaches a critical receding contact angle $\theta^{\star}\left(0 \leqslant \theta^{\star} \leqslant 1\right)$ followed by a CA phase. For a droplet evaporating in this mode, solving (3.1) yields (3.2) for $0<\tilde{t}<\tilde{t}^{\star}$ and

$$
R_{0}=\frac{\left(1+2 \theta^{\star}\right) \Delta C-4 \tilde{t}}{3 \theta^{\star} \Delta C}, \quad \theta_{0} \equiv \theta^{\star}, \quad V_{0}=\frac{\pi \theta_{0} R_{0}^{3}}{4}
$$

for $\tilde{t}^{\star}<\tilde{t}<\tilde{t}_{S S}$, where

$$
\tilde{t}^{\star}=\frac{\Delta C}{4}\left(1-\theta^{\star}\right) \quad \text { and } \quad \tilde{t}_{S S}=\frac{\Delta C}{4}\left(1+2 \theta^{\star}\right)
$$

are the depinning time (i.e. the time at which the contact angle $\theta_{0}$ reaches the critical receding angle $\theta^{\star}$ ) and the lifetime of the droplet, respectively. Both $\tilde{t}^{\star}$ and $\tilde{t}_{S S}$ are directly proportional to $\Delta C, \tilde{t}^{\star}$ is a linearly decreasing function of $\theta^{\star}$ satisfying $\tilde{t}^{\star}=\tilde{t}_{C R}$ at $\theta^{\star}=0$ and $\tilde{t}^{\star}=0$ at $\theta^{\star}=1$, and $\tilde{t}_{S S}$ is a linearly increasing function of $\theta^{\star}$ satisfying $\tilde{t}_{S S}=\tilde{t}_{C R}$ at $\theta^{\star}=0$ and $\tilde{t}_{S S}=\tilde{t}_{C A}$ at $\theta^{\star}=1$.

Figure 2 shows $(a) R_{0}$, (b) $\theta_{0}$ and $(c) V_{0}$ as functions of $\tilde{t}$ for various values of $\theta^{\star}$, including $\theta^{\star}=0$ (i.e. the CR mode given by (3.2)) and $\theta^{\star}=1$ (i.e. the CA mode given by (3.3)), and $(d) V_{0}$ as a function of $\tilde{t}$ for various values of $\Delta C$. Figure 2 illustrates that $R_{0}$ and $\theta_{0}$ are either constant or linearly decreasing functions of $\tilde{t}, V_{0}$ is first (for $0<\tilde{t}<\tilde{t}^{\star}$ ) a linearly and then (for $\tilde{t}^{\star}<\tilde{t}<\tilde{t}_{S S}$ ) a cubically decreasing function of $\tilde{t}$, and as $\theta^{*}$ increases the droplet depins earlier but has a longer lifetime. Somewhat more unexpectedly, figure $2(a)$ also illustrates that, except in the special case $\theta^{\star}=0, R_{0}=2 / 3$ at $\tilde{t}=\tilde{t}_{C R}$ irrespective of the value of the critical receding angle $\theta^{\star}$ (i.e. whatever the non-zero value of $\theta^{\star}$, the contact radius always reduces to $2 / 3$ of its initial value at $\tilde{t}=\tilde{t}_{C R}$ ).

\subsection{Evolution of a droplet evaporating in the stick-jump mode}

The stick-jump (SJ) mode of evaporation consists of an infinite series of stick (i.e. $\mathrm{CR}$ ) phases separated by an infinite series of jump phases in which the contact angle jumps instantaneously from a minimum value $\theta_{\min }$ to a maximum value $\theta_{\max }\left(0 \leqslant \theta_{\min } \leqslant\right.$ $\left.\theta_{\max } \leqslant 1\right)$ with a corresponding instantaneous jump in the contact radius. For a droplet evaporating in this mode, if we denote by $R_{n}(n=1,2,3, \ldots)$ the constant value of $R_{0}$ during the $n$th stick (i.e. CR) phase lasting from $\tilde{t}=\tilde{t}_{n-1}$ to $\tilde{t}=\tilde{t}_{n}$, then, by conservation of mass during the $n$th jump phase occurring at $\tilde{t}=\tilde{t}_{n}(n=1,2,3, \ldots)$, we have $\theta_{\min } R_{n}^{3}=\theta_{\max } R_{n+1}^{3}$, and so

$$
R_{n+1}=\left(\frac{\theta_{\min }}{\theta_{\max }}\right)^{1 / 3} R_{n}=\left(\frac{\theta_{\min }}{\theta_{\max }}\right)^{n / 3} R_{1} .
$$



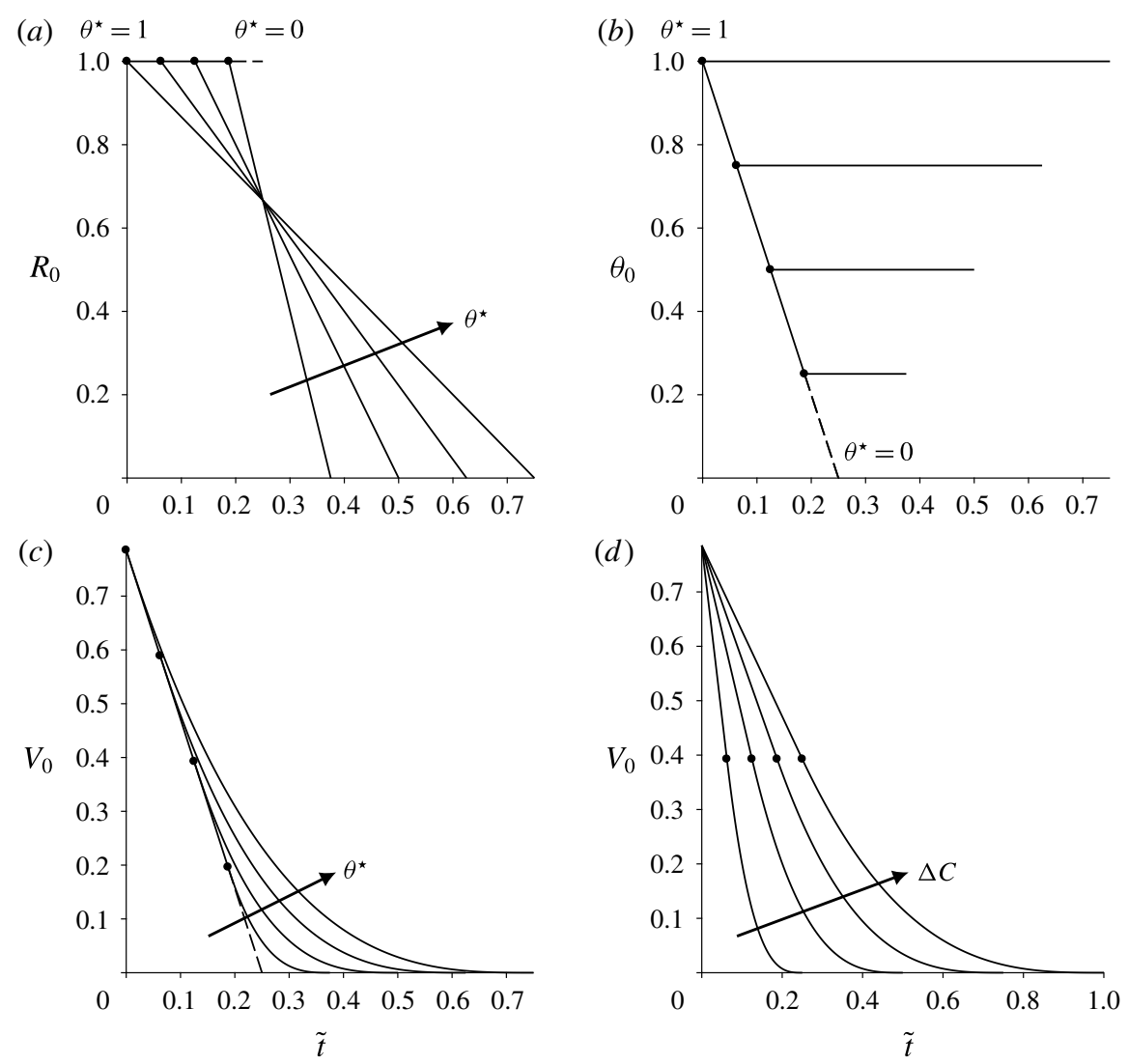

FIGURE 2. Evolution of a droplet on a substrate with a high thermal resistance (i.e. in the limit $S \rightarrow \infty$ with $\Delta C \neq 0$ ) evaporating in the SS mode. Plots of $(a) R_{0},(b) \theta_{0}$ and (c) $V_{0}$ as functions of $\tilde{t}$ for $\theta^{\star}=0$ (i.e. the CR mode, shown dashed), $1 / 4,1 / 2,3 / 4$ and 1 (i.e. the CA mode) with $\Delta C=1$, and $(d) V_{0}$ as a function of $\tilde{t}$ for $\Delta C=1 / 2,1,3 / 2$ and 2 with $\theta^{\star}=1 / 2$. The dots $(\bullet)$ denote the instants at which depinning occurs (i.e. $\left.\tilde{t}=\tilde{t}^{\star}\right)$, and in $(a),(c)$ and $(d)$ the arrows indicate the direction of increasing values of the appropriate parameter.

During the first stick phase with $R_{0}=R_{1} \equiv 1$ from $\tilde{t}=\tilde{t}_{0}=0$ to $\tilde{t}=\tilde{t}_{1}=\Delta C\left(1-\theta_{\text {min }}\right) / 4$, $\theta_{0}$ and $V_{0}$ are given by (3.2), and thereafter during the $n$th stick phase $(n=2,3,4, \ldots)$ with $R_{0}=R_{n}$ from $\tilde{t}=\tilde{t}_{n-1}$ to $\tilde{t}=\tilde{t}_{n}$,

$$
R_{0}=R_{n}, \quad \theta_{0}=\theta_{\max }-\frac{4}{\Delta C R_{n}}\left(\tilde{t}-\tilde{t}_{n-1}\right), \quad V_{0}=\frac{\pi \theta_{0} R_{n}^{3}}{4},
$$

where

$$
\tilde{t}_{n}=\frac{\Delta C}{4}\left[1-\theta_{\max }+\left(\theta_{\max }-\theta_{\min }\right) \frac{1-\left(\theta_{\min } / \theta_{\max }\right)^{n / 3}}{1-\left(\theta_{\min } / \theta_{\max }\right)^{1 / 3}}\right] .
$$

Taking the limit $n \rightarrow \infty$ in (3.8) we obtain the lifetime of the droplet, namely

$$
\tilde{t}_{S J}=\frac{\Delta C}{4}\left[1-\theta_{\max }+\left(\theta_{\max }-\theta_{\min }\right) \frac{\theta_{\max }{ }^{1 / 3}}{\theta_{\max }^{1 / 3}-\theta_{\min }^{1 / 3}}\right] .
$$


In particular, $\tilde{t}_{S J}$ is directly proportional to $\Delta C$, and is an increasing function of both $\theta_{\max }$ and $\theta_{\min }\left(<\theta_{\max }\right)$ satisfying $\tilde{t}_{S J}=\tilde{t}_{C R}$ when $\theta_{\max }=0$ and when $\theta_{\min }=0$, and $\tilde{t}_{S J} \rightarrow \tilde{t}_{S S}$ when $\theta_{\max } \rightarrow \theta_{\min }=\theta^{\star}$.

Figure 3 shows $(a) R_{0}$, (b) $\theta_{0}$ and (c) $V_{0}$ as functions of $\tilde{t}$ for various values of $\theta_{\min },(d) V_{0}$ as a function of $\tilde{t}$ for various values of $\Delta C,(e) \tilde{t}_{S J}$ as a function of $\theta_{\min }\left(\leqslant \theta_{\max }\right)$ for various values of $\theta_{\max }$, and $(f) \tilde{t}_{S J}$ as a function of $\theta_{\max }\left(\geqslant \theta_{\min }\right)$ for various values of $\theta_{\min }$. Figure 3 illustrates that $R_{0}$ is constant and $\theta_{0}$ is a linearly decreasing function of $\tilde{t}$ during each stick phase, $R_{0}$ and $\theta_{0}$ jump instantaneously down and up, respectively, during each jump phase, and as the droplet evaporates the stick phases get progressively shorter (approaching zero duration in the limit $n \rightarrow \infty$ ).

\section{When the saturation concentration depends strongly on temperature}

A similar analysis to that described in $\S 3$ can be performed when the saturation concentration of the vapour depends strongly on temperature, corresponding to the asymptotic limit $\Delta C \rightarrow \infty$.

Inspection of (2.7)-(2.15) suggests that in the limit $\Delta C \rightarrow \infty$ the complete evolution of a droplet will occur over the long time scale $t=O(\Delta C) \gg 1$. Proceeding in the same manner as in $\S 3$ we therefore rescale $c$ and $J$ with $1 / \Delta C$ and $t$ with $\Delta C$ by writing $c=\hat{c} / \Delta C, J=\hat{J} / \Delta C$ and $t=\Delta C \hat{t}$, and seek an asymptotic expansion for $\hat{c}$ in the form $\hat{c}(r, z, \hat{t})=\hat{c}_{0}(r, z, \hat{t})+O(1 / \Delta C)$ with corresponding asymptotic expansions for the other dependent variables.

In the limit $\Delta C \rightarrow \infty$ we obtain

$$
\begin{gathered}
\hat{J}=\frac{2 R_{0}}{\left[\theta_{0}\left(R_{0}^{2}-r^{2}\right)+2 S R_{0}\right] \Delta C}+O\left(\frac{1}{\Delta C^{2}}\right), \\
T=1-\frac{2 E R_{0}(z+S)}{\left[\theta_{0}\left(R_{0}^{2}-r^{2}\right)+2 S R_{0}\right] \Delta C}+O\left(\frac{1}{\Delta C^{2}}\right), \\
T^{s}=1-\frac{2 E R_{0} S(z+1)}{\left[\theta_{0}\left(R_{0}^{2}-r^{2}\right)+2 S R_{0}\right] \Delta C}+O\left(\frac{1}{\Delta C^{2}}\right),
\end{gathered}
$$

and hence (2.15) yields the equation describing the leading-order evolution of the droplet, namely

$$
\frac{\mathrm{d}}{\mathrm{d} \hat{t}}\left(\theta_{0} R_{0}^{3}\right)=-\frac{8 R_{0}}{\theta_{0}} \log \left(\frac{2 S+\theta_{0} R_{0}}{2 S}\right) .
$$

In contrast to the limit $S \rightarrow \infty$, in this limit we are unable to obtain an explicit expression for the leading-order concentration of vapour, $\hat{c}_{0}$, but, as in the limit $S \rightarrow \infty$, this is not required in order to determine the leading-order evolution of the droplet.

\subsection{Evolution of a droplet evaporating in the constant radius mode} A droplet evaporating in the CR mode satisfies

$$
R_{0} \equiv 1, \quad \hat{t}=F(1,1, S)-F\left(1, \theta_{0}, S\right), \quad V_{0}=\frac{\pi \theta_{0}}{4},
$$

and hence $\hat{t}_{C R}=F(1,1, S)$, where $F\left(R_{0}, \theta_{0}, S\right)$ is an increasing function of each of its three arguments defined by

$$
F\left(R_{0}, \theta_{0}, S\right)=\frac{S^{2}}{2}\left[\operatorname{Ei}\left(2 \log \left(\frac{2 S+\theta_{0} R_{0}}{2 S}\right)\right)-\mathrm{Ei}\left(\log \left(\frac{2 S+\theta_{0} R_{0}}{2 S}\right)\right)-\log 2\right],
$$



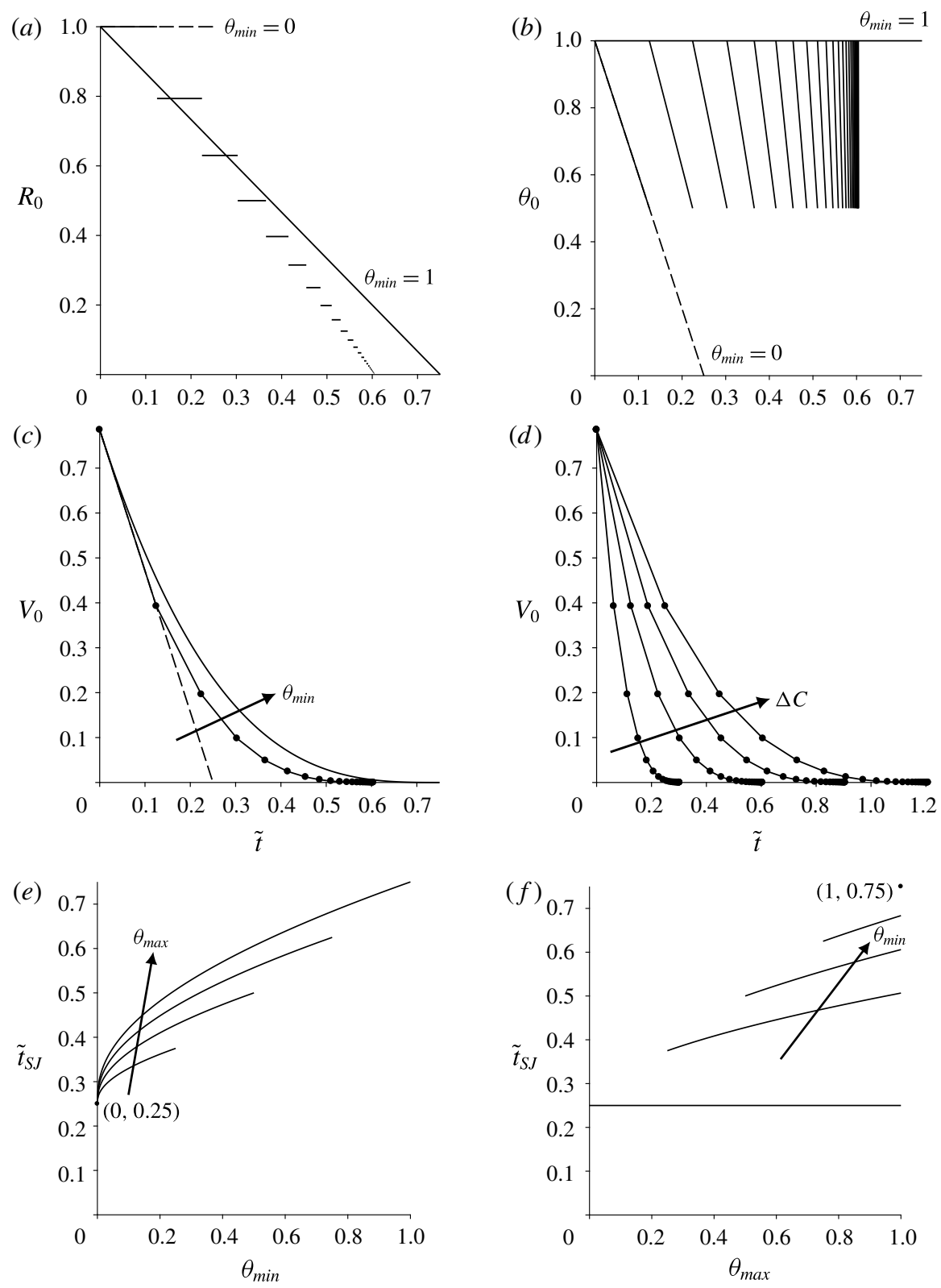

FIGURE 3. Evolution of a droplet on a substrate with a high thermal resistance (i.e. in the limit $S \rightarrow \infty$ with $\Delta C \neq 0$ ) evaporating in the SJ mode. Plots of $(a) R_{0},(b) \theta_{0}$ and (c) $V_{0}$ as functions of $\tilde{t}$ for $\theta_{\min }=0$ (i.e. the CR mode, shown dashed), $1 / 2$ and 1 (i.e. the CA mode) with $\theta_{\max }=1$ and $\Delta C=1,(d) V_{0}$ as a function of $\tilde{t}$ for $\Delta C=1 / 2,1$, $3 / 2$ and 2 with $\theta_{\max }=1$ and $\theta_{\min }=1 / 2,(e) \tilde{t}_{S J}$ as a function of $\theta_{\min }\left(\leqslant \theta_{\max }\right)$ for $\theta_{\max }=0$ (i.e. the CR mode), $1 / 4,1 / 2,3 / 4$ and 1 , and $(f) \tilde{t}_{S J}$ as a function of $\theta_{\max }\left(\geqslant \theta_{\min }\right)$ for $\theta_{\min }=0$ (i.e. the $\mathrm{CR}$ mode), $1 / 4,1 / 2,3 / 4$ and 1 (i.e. the CA mode), with $\Delta C=1$. In $(c)$ and $(d)$ the dots $(\bullet)$ denote the instants at which the jump phases occur (i.e. $\tilde{t}=\tilde{t}_{n}$ for $n=1,2,3, \ldots)$, and in $(c)-(f)$ the arrows indicate the direction of increasing values of the appropriate parameter. 
in which $\operatorname{Ei}(\cdot)$ denotes the exponential integral

$$
\operatorname{Ei}(\xi)=-\int_{-\xi}^{\infty} \frac{\mathrm{e}^{-\lambda}}{\lambda} \mathrm{d} \lambda
$$

Note that $F\left(R_{0}, \theta_{0}, S\right)$ takes the value zero when any one of its arguments is zero.

\subsection{Evolution of a droplet evaporating in the constant angle mode}

A droplet evaporating in the CA mode satisfies

$$
\hat{t}=3\left[F(1,1, S)-F\left(R_{0}, 1, S\right)\right], \quad \theta_{0} \equiv 1, \quad V_{0}=\frac{\pi R_{0}^{3}}{4},
$$

and hence $\hat{t}_{C A}=3 F(1,1, S)=3 \hat{t}_{C R}$.

Both $\hat{t}_{C R}$ and $\hat{t}_{C A}$ are increasing functions of $S$ satisfying $\hat{t}_{C R}, \hat{t}_{C A}=O(1 / \log S) \rightarrow 0^{+}$ as $S \rightarrow 0^{+}$, and $\hat{t}_{C R} \sim S / 4 \rightarrow \infty$ and $\hat{t}_{C A} \sim 3 S / 4 \rightarrow \infty$ as $S \rightarrow \infty$, and (as in the limit $S \rightarrow \infty$ ) their ratio is exactly 3 .

The solutions in the limit $\Delta C \rightarrow \infty$ given by (4.5) and (4.8) are similar, but not identical, to the corresponding solutions in the limit $S \rightarrow \infty$ given by (3.2) and (3.3) with $S$ replaced by $\Delta C$. Unlike the corresponding solutions in the limit $S \rightarrow \infty, \theta_{0}$ and $R_{0}$ are not simply linear functions of $\hat{t}$ in the CR and CA modes, respectively, and $\hat{t}_{C R}$ and $\hat{t}_{C A}$ are not simply linear functions of $S$. However, except for small values of $S$, the nonlinear function $F\left(R_{0}, \theta_{0}, S\right)$ defined by (4.6) is very well approximated by its linear leading-order small $R_{0}$ and/or small $\theta_{0}$ and/or large $S$ behaviour, i.e. $F\left(R_{0}, \theta_{0}, S\right) \approx$ $\theta_{0} R_{0} S / 4$. Hence, except for small values of $S$, the solutions in the limit $\Delta C \rightarrow \infty$ are very well approximated by the corresponding solutions in the limit $S \rightarrow \infty$ with $S$ replaced by $\Delta C$. In particular, except for small values of $S, \hat{t}_{C R} \approx S / 4$ and $\hat{t}_{C A} \approx 3 S / 4$.

\subsection{Evolution of a droplet evaporating in the stick-slide mode}

A droplet evaporating in the SS mode satisfies (4.5) for $0<\tilde{t}<\tilde{t}^{\star}$ and

$$
\hat{t}=2 F\left(1, \theta^{\star}, S\right)+F(1,1, S)-3 F\left(R_{0}, \theta^{\star}, S\right), \quad \theta_{0} \equiv \theta^{\star}, \quad V_{0}=\frac{\pi \theta^{\star} R_{0}^{3}}{4},
$$

for $\hat{t}^{\star}<\hat{t}<\hat{t}_{S S}$, where

$$
\hat{t}^{\star}=F(1,1, S)-F\left(1, \theta^{\star}, S\right) \quad \text { and } \quad \hat{t}_{S S}=2 F\left(1, \theta^{\star}, S\right)+F(1,1, S) .
$$

Both $\hat{t}^{\star}$ and $\hat{t}_{S S}$ are increasing functions of $S$ satisfying $\hat{t}^{\star}, \hat{t}_{S S}=O(1 / \log S) \rightarrow 0^{+}$ as $S \rightarrow 0^{+}$, and $\hat{t}^{\star} \sim S\left(1-\theta^{\star}\right) / 4 \rightarrow \infty$ and $\hat{t}_{S S} \sim S\left(1+2 \theta^{\star}\right) / 4 \rightarrow \infty$ as $S \rightarrow \infty$. Furthermore, $\hat{t}^{\star}$ is a decreasing function of $\theta^{\star}$ satisfying $\hat{t}^{\star}=\hat{t}_{C R}$ at $\theta^{\star}=0$ and $\hat{t}^{\star}=0$ at $\theta^{\star}=1$, whereas $\hat{t}_{S S}$ is an increasing function of $\theta^{\star}$ satisfying $\hat{t}_{S S}=\hat{t}_{C R}$ at $\theta^{\star}=0$ and $\hat{t}_{S S}=\hat{t}_{C A}$ at $\theta^{\star}=1$. As for a droplet evaporating in either the CR or the CA mode, except for small values of $S$, the solutions in the limit $\Delta C \rightarrow \infty$ are again very well approximated by the corresponding solutions in the limit $S \rightarrow \infty$ with $S$ replaced by $\Delta C$, and so require no further discussion here. 


\subsection{Evolution of a droplet evaporating in the stick-jump mode}

A droplet evaporating in the SJ mode again satisfies (3.6). During the 1st stick phase with $R_{0}=R_{1} \equiv 1$ from $\hat{t}=\hat{t}_{0}=0$ to $\hat{t}=\hat{t}_{1}=F(1,1, S)-F\left(1, \theta_{\min }, S\right), \theta_{0}$ and $V_{0}$ are given by (4.5), and thereafter during the $n$th stick phase $(n=2,3,4, \ldots)$ with $R_{0}=R_{n}$ from $\hat{t}=\hat{t}_{n-1}$ to $\hat{t}=\hat{t}_{n}$,

$$
R_{0}=R_{n}, \quad \hat{t}-\hat{t}_{n-1}=F\left(R_{n}, \theta_{\max }, S\right)-F\left(R_{n}, \theta_{0}, S\right), \quad V_{0}=\frac{\pi \theta_{0} R_{n}^{3}}{4}, \quad(4.11 a-c)
$$

where

$$
\hat{t}_{n}=F(1,1, S)-F\left(1, \theta_{\min }, S\right)+\sum_{m=2}^{n} F\left(R_{m}, \theta_{\max }, S\right)-F\left(R_{m}, \theta_{\min }, S\right) .
$$

Taking the limit $n \rightarrow \infty$ in (4.12) we obtain the lifetime of the droplet, namely

$$
\hat{t}_{S J}=F(1,1, S)-F\left(1, \theta_{\min }, S\right)+\sum_{m=2}^{\infty} F\left(R_{m}, \theta_{\max }, S\right)-F\left(R_{m}, \theta_{\min }, S\right) .
$$

In particular, $\hat{t}_{S J}$ is an increasing function of $S$ satisfying $\hat{t}_{S J} \rightarrow 0^{+}$as $S \rightarrow 0^{+}$, and $\hat{t}_{S J}=O(S) \rightarrow \infty$ as $S \rightarrow \infty$. Furthermore, $\hat{t}_{S J}$ is an increasing function of both $\theta_{\max }$ and $\theta_{\min }\left(<\theta_{\max }\right)$ satisfying $\hat{t}_{S J}=\hat{t}_{C R}$ when $\theta_{\max }=0$ and when $\theta_{\min }=0$, and $\hat{t}_{S J} \rightarrow \hat{t}_{S S}$ when $\theta_{\max } \rightarrow \theta_{\min }=\theta^{\star}$. As for a droplet evaporating in either the CR or the CA mode, except for small values of $S$, the solutions in the limit $\Delta C \rightarrow \infty$ are again very well approximated by the corresponding solutions in the limit $S \rightarrow \infty$ with $S$ replaced by $\Delta C$, and so again require no further discussion here.

\section{Conclusions}

In the present work we have analysed the evaporation of a thin sessile droplet on a thin substrate in two situations in which the influence of the thermal properties of the system is strong. Specifically, we have obtained uniformly valid leading-order asymptotic solutions for the evolution of the droplet when the substrate has a high thermal resistance relative to the droplet (corresponding to the limit $S \rightarrow \infty$ with $\Delta C \neq 0$ ) and when the saturation concentration of the vapour depends strongly on temperature (corresponding to the limit $\Delta C \rightarrow \infty$ ). In both situations we have obtained explicit expressions for the lifetimes of the droplet for all four of the modes of evaporation studied in the present work (namely the CR, CA, SS and SJ modes).

The basic model, which is applicable when the influence of the thermal properties of the system on the evolution of the droplet is weak, predicts that the lifetimes of the droplet are of the order of the basic time scale (2.2). In contrast, the present work shows that when the influence of the thermal properties of the system on the evolution of the droplet is strong (specifically, in the limit $S \rightarrow \infty$ and to a very good approximation in the limit $\Delta C \rightarrow \infty$ ), the lifetimes of the droplet are much longer than the basic time scale by a factor of size $S \Delta C \gg 1$, i.e. are actually on the much longer time scale

$$
\frac{\rho \theta(0) R(0) \mathcal{L} h^{s} c_{\text {sat }}^{\prime}\left(T_{\infty}\right)}{k^{s}(1-H) c_{\text {sat }}\left(T_{\infty}\right)}
$$

The different dependence of the time scales given by (2.2) and (5.1) on the physical parameters reflects the different dominant physical mechanisms when thermal effects are weak and when they are strong. The basic time scale (2.2), which is independent 


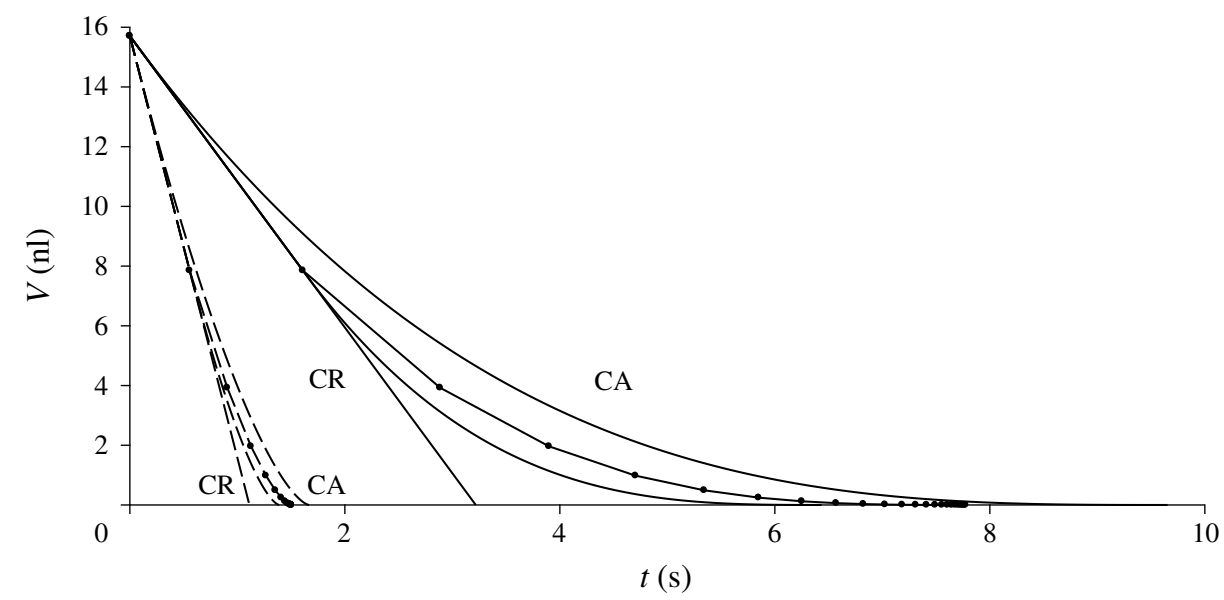

FIgURE 4. Evolution of the dimensional volume $V$ (in $\mathrm{nl}$ ) as a function of dimensional time $t$ (in s) for a thin droplet of methanol on a substrate made of a good thermal conductor according to the basic model (dashed curves) and on a thin substrate made of an aerogel according to the present leading-order solution in the limit $S \rightarrow \infty$ with $\Delta C \neq 0$ (solid curves). In both cases the four curves correspond (from left to right) to evaporation in the CR, SS, SJ and CA modes. The dots (๑) denote the instants at which depinning (SS mode) and the jump phases (SJ mode) occur.

of $\mathcal{L}, k, k^{s}$ and $h^{s}$ (i.e. independent of the thermal properties of the system and the thickness of the substrate), corresponds to the familiar situation described by the basic diffusion-limited model in which the evaporation from the droplet is limited by diffusion of vapour in the atmosphere with constant saturation concentration at the free surface of the droplet. In contrast, the time scale (5.1), which is independent of $k$ and $D$ (i.e. independent of the thermal conductivity of the droplet and the coefficient of diffusion of vapour in the atmosphere), corresponds to the situation in which the evaporation from the droplet is limited by thermal conduction through the droplet and the substrate.

To illustrate the difference between the two situations, consider a thin droplet of methanol with initial radius $R(0)=10^{-3} \mathrm{~m}$ and initial contact angle $\theta(0)=0.02$ in an atmosphere of air with $H=0$, for which, using the typical parameter values given by Dunn et al. $(2009 a), \rho=790 \mathrm{~kg} \mathrm{~m}^{-3}, \mathcal{L}=1.20 \times 10^{6} \mathrm{~m}^{2} \mathrm{~s}^{-2}, k=$ $0.203 \mathrm{~kg} \mathrm{~m} \mathrm{~s}^{-3} \mathrm{~K}^{-1}, c_{\text {sat }}\left(T_{\infty}\right)=0.186 \mathrm{~kg} \mathrm{~m}^{-3}, c_{\text {sat }}^{\prime}\left(T_{\infty}\right)=9.47 \times 10^{-3} \mathrm{~kg} \mathrm{~m}^{-3} \mathrm{~K}^{-1}$ at $T_{\infty}=295 \mathrm{~K}$ and $D=1.50 \times 10^{-5} \mathrm{~m}^{2} \mathrm{~s}^{-1}$. The basic model predicts that on a substrate made of a good thermal conductor (such as, for example, a metal) the droplet evaporates completely in between 1.11 and $1.67 \mathrm{~s}$ (corresponding to the CR and CA modes, respectively). On the other hand, the present leading-order solution in the limit $S \rightarrow \infty$ with $\Delta C \neq 0$ predicts that on a thin substrate of thickness $h^{s}=2 \times 10^{-4} \mathrm{~m}$ made of a poor thermal conductor, specifically an aerogel with a typical thermal conductivity of $k^{s}=0.015 \mathrm{~kg} \mathrm{~m} \mathrm{~s}^{-3} \mathrm{~K}^{-1}$ (see, for example, Cohen \& Glicksman 2015), corresponding to $\Delta C=0.017$ and $S=135$, the same droplet evaporates completely in between 3.22 and 9.65 s. Figure 4 shows the evolution of the dimensional volume $V$ of the droplet as a function of dimensional time $t$ for both substrates for all four modes of evaporation. In particular, figure 4 illustrates the main conclusion of the present work, namely that when thermal effects are strong 
the lifetimes of evaporating droplets are significantly extended relative to those when thermal effects are weak.

We are unaware of any previous physical experiments against which the present theoretical predictions can be validated, but hope that our work will inspire one or more of the many experimental groups with an interest in evaporating droplets to conduct such experiments in the future.

Although, in order to permit analytical progress, the present analysis was restricted to the case of a thin droplet on a thin substrate, we would anticipate that qualitatively similar behaviour will occur for more general droplets on more general substrates.

\section{Acknowledgements}

F.G.H.S. acknowledges the financial support of the United Kingdom Engineering and Physical Sciences Research Council (EPSRC) via EPSRC Doctoral Training Partnership grant EP/N509760/1, the University of Strathclyde, and the University of Edinburgh. K.S. acknowledges financial support from the EVAPORATION Microgravity Application Programme project of the European Space Agency (ESA) (contract number 4200014293). The authors acknowledge valuable discussions with Dr B. R. Duffy and Dr A. W. Wray (University of Strathclyde) on various aspects of droplet evaporation. The authors also wish to thank all three anonymous referees for their insightful comments on the original version of this work.

\section{REFERENCES}

Ait SAadA, M., Chikh, S. \& TAdrist, L. 2013 Evaporation of a sessile drop with pinned or receding contact line on a substrate with different thermophysical properties. Intl J. Heat Mass Transfer 58, 197-208.

Askounis, A., Orejon, D., Koutsos, V., Sefiane, K. \& Shanahan, M. E. R. 2011 Nanoparticle deposits near the contact line of pinned volatile droplets: size and shape revealed by atomic force microscopy. Soft Matt. 7, 4152-4155.

Cazabat, A.-M. \& GuÉnA, G. 2010 Evaporation of macroscopic sessile droplets. Soft Matt. 6, 2591-2612.

Cohen, E. \& Glicksman, L. 2015 Thermal properties of silica aerogel formula. Trans. ASME J. Heat Transfer 137, 081601.

DAsh, S. \& GARIMELla, S. V. 2013 Droplet evaporation dynamics on a superhydrophobic surface with negligible hysteresis. Langmuir 29, 10785-10795.

Diddens, C., Tan, H., Lv, P., Versluis, M., Kuerten, J. G. M., Zhang, X. \& Lohse, D. 2017 Evaporating pure, binary and ternary droplets: thermal effects and axial symmetry breaking. J. Fluid Mech. 823, 470-497.

Dietrich, E., Kooij, E. S., Zhang, X., Zandvliet, H. J. W. \& Lohse, D. 2015 Stick-jump mode in surface droplet dissolution. Langmuir 31, 4696-4703.

Dunn, G. J., Wilson, S. K., Duffy, B. R., David, S. \& Sefiane, K. 2008 A mathematical model of the evaporation of a thin sessile liquid droplet: comparison between experiment and theory. Colloids Surf. A 323, 50-55.

Dunn, G. J., Wilson, S. K., Duffy, B. R., David, S. \& Sefiane, K. $2009 a$ The strong influence of substrate conductivity on droplet evaporation. J. Fluid Mech. 623, 329-351.

Dunn, G. J., Wilson, S. K., Duffy, B. R. \& Sefiane, K. $2009 b$ Evaporation of a thin droplet on a thin substrate with a high thermal resistance. Phys. Fluids 21, 052101.

ErbiL, H. Y. 2012 Evaporation of pure liquid sessile and spherical suspended drops: a review. Adv. Colloid Interface Sci. 170, 67-86.

LARSON, R. G. 2014 Transport and deposition patterns in drying sessile droplets. AIChE J. 60, $1538-1571$. 
Lohse, D. \& Zhang, X. 2015 Surface nanobubbles and nanodroplets. Rev. Mod. Phys. 87, 981-1035.

Lopes, M. C., Bonaccurso, E., Gambaryan-Roisman, T. \& Stephan, P. 2013 Influence of the substrate thermal properties on sessile droplet evaporation: effect of transient heat transport. Colloids Surf. A 432, 64-70.

Menta, R. C. \& Bose, T. K. 1983 Temperature distribution on a large circular plate heated by a disk heat source. Intl J. Heat Mass Transfer 27, 1093-1095.

Nguyen, T. A. H. \& NGuYen, A. V. 2012 Increased evaporation kinetics of sessile droplets by using nanoparticles. Langmuir 28, 16725-16728.

Orejon, D., Sefiane, K. \& Shanahan, M. E. R. 2011 Stick-slip of evaporating droplets: substrate hydrophobicity and nanoparticle concentration. Langmuir 27, 12834-12843.

Picknett, R. G. \& BEXON, R. 1977 The evaporation of sessile or pendant drops in still air. J. Colloid Interface Sci. 61, 336-350.

Popov, Y. O. 2005 Evaporative deposition patterns: spatial dimensions of the deposit. Phys. Rev. E 71, 036313.

SEFIANE, K. \& BenNACER, R. 2011 An expression for droplet evaporation incorporating thermal effects. J. Fluid Mech. 667, 260-271.

Sefiane, K., Wilson, S. K., David, S., Dunn, G. J. \& Duffy, B. R. 2009 On the effect of the atmosphere on the evaporation of sessile droplets of water. Phys. Fluids 21, 062101.

Sobac, B. \& Brutin, D. 2012 Thermal effects of the substrate on water droplet evaporation. Phys. Rev. E 86, 021602.

Stauber, J. M. 2015 On the evaporation of sessile droplets. PhD thesis, University of Strathclyde, Glasgow.

Stauber, J. M., Wilson, S. K., Duffy, B. R. \& Sefiane, K. 2014 On the lifetimes of evaporating droplets. J. Fluid Mech. 744, R2.

Stauber, J. M., Wilson, S. K., Duffy, B. R. \& Sefiane, K. 2015 On the lifetimes of evaporating droplets with related initial and receding contact angles. Phys. Fluids 27, 122101. 